\title{
SPARSE COMPLEX FXLMS FOR ACTIVE NOISE CANCELLATION OVER SPATIAL REGIONS
}

\author{
Jihui Zhang ${ }^{\dagger *}$, Thushara D. Abhayapala*, Prasanga N. Samarasinghe*, Wen Zhang*, Shouda Jiang ${ }^{\dagger}$ \\ ${ }^{\dagger}$ School of Electrical Engineering and Automation, Harbin Institute of Technology, \\ Harbin 150001, China \\ * Research School of Engineering, The Australian National University, \\ Canberra ACT 2601, Australia
}

\begin{abstract}
In this paper, we investigate active noise control over large $2 \mathrm{D}$ spatial regions when the noise source is sparsely distributed. The $\ell_{1}$ relaxation technique originated from compressive sensing is adopted and based on that we develop the algorithm for two cases: multipoint noise cancellation and wave domain noise cancellation. This results in two new variants (i) zero-attracting multi-point complex FxLMS and (ii) zero-attracting wave domain complex FxLMS. Both approaches use a feedback control system, where a microphone array is distributed over the boundary of the control region to measure the residual noise signals and a loudspeaker array is placed outside the microphone array to generate the anti-noise signals. Simulation results demonstrate the performance and advantages of the proposed methods in terms of convergence rate and spatial noise reduction levels.
\end{abstract}

Index Terms - Active noise control, Multi-channel system, Adaptive algorithm, Compressive sampling, Wave domain

\section{INTRODUCTION}

Active noise control (ANC), or noise cancellation, involves an electroacoustic or electromechanical system that employs secondary sound sources to reduce the primary noise based on the principle of destructive interference [1,2,3]. This technique has been successfully used in several commercial applications, such as noisecancelling headphones $[4,5]$, the control of noise in industrial machines [6] and active noise cancellation for automobiles [7, 8, 9]. Given the fact that most of the time, noise is unknown and timevarying, adaptive filters are employed to produce anti-noise signals. Some well-known algorithms for implementation include the leastmean-square (LMS) method or its variants, such as filtered-x LMS (FxLMS) [10], adjoint LMS [11] and recursive LMS [12].

Noise cancellation over a few observation points is simple and effective, where only one or two microphones and speakers are required to measure the residual signal and to produce the anti-noise signal. A growing research interest is in expanding the above solution to multiple listening points, which in turn requires increased numbers of sensor and source units, and is named as multi-channel ANC systems. There exist two main signal processing techniques for multi-channel ANC systems. The first technique does noise cancel-

This work is supported by Australian Research Council (ARC) Discovery Projects funding scheme (project no. DP140103412). The work of J. Zhang was sponsored by the China Scholarship Council with the Australian National University. lation directly on a set of multiple observation points (MP) ${ }^{1}$ in the control region [13], whereas the second or the more recent technique does wave domain (WD) noise cancellation over the entire continuous control region [14]. The MP approach is fairly straightforward and therefore is widely used in practice $[15,16,17]$. In contrast, the WD approach is slightly advanced as it exploits the wave domain signal representation of the Helmhotz wave equation to control the overall continuous noise region $[18,19]$. Recent work in wave domain adaptive ANC include feed-forward systems [14] as well as feed-back systems [20].

While the above techniques are capable of achieving significant noise cancellation over spatial regions, their respective adaptive algorithms could be inefficient, especially when the noise field is sparse. In typical applications such as in-car noise cancellation, this sparse characteristic is very common [21, 22]. Note that sparsity is a preferred characteristic in compressive sensing to bring down the sensor/transducer numbers as well as the computational complexity of signal processing algorithms. The $\ell_{1}$-norm penalty is currently a popular compressive sensing solution of this nature [23], and could be adopted in the present ANC problem to improve the system performance.

In this paper, we design new complex FxLMS algorithms combined with the $\ell_{1}$-norm constraint to assist adaptive ANC in sparse noise fields. The new approaches are expected to improve the algorithm efficiency by introducing a constraint on the loudspeaker weights in the secondary source array. We develop the solutions for both multi-point noise cancellation as well as wave domain noise cancellation resulting in two new algorithms (i) zero-attracting multi-point complex FxLMS (ZA-MP-CFxLMS), and (ii) zeroattracting wave domain complex FxLMS (ZA-WD-CFxLMS). The proposed ZA-MP-CFxLMS algorithm can be generalized for other sparse CFxLMS algorithms, and the proposed ZA-WD-CFxLMS algorithm can be generalized for other transfer domain (TD) sparse CFxLMS algorithms. These are novel additions to the existing theory as current sparsity solutions are limited to their non-complex variants $[24,25]$.

The rest of this paper is organized as follows. The ZA-MPCFxLMS algorihm for noise cancellation is proposed in Section 2. In Section 3, we derive the ZA-WD-CFxLMS algorithm for wave domain noise cancellation. In Section 4, simulations are conducted to evaluate the proposed algorithm in comparison with the conventional multi-channel ANC system, while the last section gives some concluding remarks.

\footnotetext{
${ }^{1}$ Here, the noise cancellation happens essentially at the measurement or observation points and a small region around these points.
} 
Notation: $(\cdot)^{*}$ and $(\cdot)^{T}$ donate the conjugate and transpose operators, respectively. $\Re(\cdot)$ and $\Im(\cdot)$ denote the real part and the imaginary part of a complex quantity, respectively. The operator $\|\cdot\|_{1}$ denotes $\ell_{1}$-norm. $\exp (\cdot)$ denotes the exponential function.

\section{MULTI-POINT NOISE CANCELLATION USING THE ZA-MP-CFXLMS ALGORITHM}

In this section, we develop a zero-attracting complex FxLMS algorithm for multi-point noise cancellation. Consider an adaptive ANC system with a $P$-point microphone array (2D) and a $Q$-point loudspeaker array (2D) in free field conditions.

The frequency domain measurement at the microphone array can be represented by

$$
\boldsymbol{e}(n)=\boldsymbol{\nu}(n)+\boldsymbol{s}(n)
$$

where $\boldsymbol{e}$ is the residual noise field, $\boldsymbol{\nu}$ is the original noise field, $\boldsymbol{s}$ is the anti-noise generated by the loudspeaker array and $n$ is the iteration index of the adaptive algorithm. Note that all components of (1) are $P$-long vectors and the frequency dependence of each vector is ignored for notational simplicity. Assuming the updated weights are $\boldsymbol{w}(n)$, an FxLMS type formulation [17] is

$$
\boldsymbol{e}(n)=\boldsymbol{\nu}(n)+\boldsymbol{G} \boldsymbol{w}(n)
$$

where $\boldsymbol{G}$ is a transfer function and the initial filter weights are denoted as $\boldsymbol{w}(0)$.

In the current multi-point system with $Q$ loudspeakers, we directly populate $\boldsymbol{w}(n)$ with the loudspeaker weights $\boldsymbol{d}(n)=$ $\left[d_{1}, \cdots, d_{Q}\right]$ by setting $\boldsymbol{w}(n)=\boldsymbol{d}(n)$ such that $\boldsymbol{G}$ becomes the point source propagation characterization

$$
\boldsymbol{G}=\frac{i}{4}\left[\begin{array}{ccc}
H_{0}^{(1)}\left(k\left\|\boldsymbol{z}_{1}-\boldsymbol{\chi}_{1}\right\|\right) & \cdots & H_{0}^{(1)}\left(k\left\|\boldsymbol{z}_{Q}-\boldsymbol{\chi}_{1}\right\|\right) \\
\vdots & \ddots & \vdots \\
H_{0}^{(1)}\left(k\left\|\boldsymbol{z}_{1}-\boldsymbol{\chi}_{P}\right\|\right) & \cdots & H_{0}^{(1)}\left(k\left\|\boldsymbol{z}_{Q}-\boldsymbol{\chi}_{P}\right\|\right)
\end{array}\right]
$$

where $\left\{\left(\left\|\chi_{1}\right\|, \theta_{1}\right), \cdots,\left(\left\|\chi_{P}\right\|, \theta_{P}\right)\right\}$ represent the microphone locations and $\left\{\left(\left\|\boldsymbol{z}_{1}\right\|, \phi_{1}\right), \cdots,\left(\left\|\boldsymbol{z}_{Q}\right\|, \phi_{Q}\right)\right\}$ represent the loudspeaker locations.

The goal of the adaptive ANC system is to design the loudspeaker weights to cancel the noise over the control region. While the conventional FxLMS approach is to minimize the power of the residual signal, we aim to introduce an additional $\ell_{1}$-norm constraint on the speaker weights to exploit the sparse nature of the noise distribution [25]. To achieve this, we define a new cost function

$$
\boldsymbol{\xi}(n)=\frac{1}{2} \boldsymbol{e}(n)^{2}+\lambda\|\boldsymbol{d}(n)\|_{1},
$$

where $\lambda$ is a controllable parameter to determine the degree of zero attraction for the adaptive filter coefficients [26]. Notice that both $e$ and $\boldsymbol{d}$ are complex vectors in the frequency domain, hence we name the new adaptive algorithm as a ZA-MP-CFxLMS algorithm.

Next, we derive the updating expression to satisfy the new cost function. Adopting the gradient descent algorithm, $\boldsymbol{w}(n)$ in every iteration is given by

$$
\boldsymbol{w}(n+1)=\boldsymbol{w}(n)-\frac{\mu}{2} \nabla \boldsymbol{\xi}(n)
$$

where $\mu$ is the step size. The gradient term in (5) can be written in terms of (4) as

$$
\nabla \boldsymbol{\xi}(n)=\underbrace{\nabla \frac{1}{2} \boldsymbol{e}(n)^{2}}_{\nabla \boldsymbol{\xi}_{1}}+\underbrace{\nabla \lambda\|\boldsymbol{d}(n)\|_{1}}_{\nabla \boldsymbol{\xi}_{2}} .
$$

By the complex LMS algorithm [27], the term $\nabla \boldsymbol{\xi}_{1}$ of (6) is simply $\nabla \boldsymbol{\xi}_{1}=\boldsymbol{G}^{*} \boldsymbol{e}$. To find $\nabla \boldsymbol{\xi}_{2}$ of (6), we use its relationship to the conjugate derivative [28]

$$
\nabla \boldsymbol{\xi}_{2}=2 \frac{\partial \boldsymbol{\xi}_{2}}{\partial \boldsymbol{w}^{*}}
$$

and solve for it as follows. By definition, $\|\boldsymbol{d}\|_{1}=\sum_{q=1}^{Q}\left|d_{q}\right|$, and

$$
\left|d_{q}\right|=\sqrt{\Re\left(d_{q}\right)^{2}+\Im\left(d_{q}\right)^{2}} \leq\left(\left|\Re\left(d_{q}\right)\right|+\left|\Im\left(d_{q}\right)\right|\right) .
$$

$$
\begin{aligned}
& \text { Hence, }\|\boldsymbol{d}\|_{1} \leq \sum_{q=1}^{Q}\left(\left|\Re\left(d_{q}\right)\right|+\left|\Im\left(d_{q}\right)\right|\right) \\
& =\sum_{q=1}^{Q}\left(\left|\Re\left(d_{q}\right)\right|\right)+\sum_{q=1}^{Q}\left(\left|\Im\left(d_{q}\right)\right|\right)=\|\Re(\boldsymbol{d})\|_{1}+\|\Im(\boldsymbol{d})\|_{1} .
\end{aligned}
$$

From (8), $\left(\partial \boldsymbol{\xi}_{2} / \partial \boldsymbol{w}^{*}\right)$ can be approximated by

$$
\frac{\partial \boldsymbol{\xi}_{2}}{\partial \boldsymbol{w}^{*}}=\lambda\left(\frac{\partial\|\Re(\boldsymbol{d})\|_{1}}{\partial \boldsymbol{w}^{*}}+\frac{\partial\|\Im(\boldsymbol{d})\|_{1}}{\partial \boldsymbol{w}^{*}}\right) .
$$

If the complex $\boldsymbol{w}$ is decomposed into its real and imaginary parts as $\boldsymbol{w}=\boldsymbol{x}+i \boldsymbol{y}$, the complex partial differentiation based on $\boldsymbol{w}^{*}$ can be separated [29] by

$$
\begin{gathered}
\frac{\partial\|\Re(\boldsymbol{d})\|_{1}}{\partial \boldsymbol{w}^{*}}=\frac{1}{2}\left(\frac{\partial\|\Re(\boldsymbol{d})\|_{1}}{\partial \boldsymbol{x}}+i * \frac{\partial\|\Re(\boldsymbol{d})\|_{1}}{\partial \boldsymbol{y}}\right) \\
\frac{\partial\|\Im(\boldsymbol{d})\|_{1}}{\partial \boldsymbol{w}^{*}}=\frac{1}{2}\left(\frac{\partial\|\Im(\boldsymbol{d})\|_{1}}{\partial \boldsymbol{x}}+i * \frac{\partial\|\Im(\boldsymbol{d})\|_{1}}{\partial \boldsymbol{y}}\right) .
\end{gathered}
$$

Since $\boldsymbol{d}(n)=\boldsymbol{w}(n)$, each item in the RHS of (10) can be given by

$$
\begin{gathered}
\frac{\partial\|\Re(\boldsymbol{d})\|_{1}}{\partial \boldsymbol{x}}=\operatorname{sign}(\Re(\boldsymbol{w})), \\
\frac{\partial\|\Re(\boldsymbol{d})\|_{1}}{\partial \boldsymbol{y}}=\frac{\partial\|\Im(\boldsymbol{d})\|_{1}}{\partial \boldsymbol{x}}=0, \\
\frac{\partial\|\Im(\boldsymbol{d})\|_{1}}{\partial \boldsymbol{y}}=\operatorname{sign}(\Im(\boldsymbol{w})) .
\end{gathered}
$$

Substituting (10) and (11) into (9), $\partial \boldsymbol{\xi}_{2} / \partial \boldsymbol{w}^{*}$ becomes

$$
\frac{\partial \boldsymbol{\xi}_{2}}{\partial \boldsymbol{w}^{*}}=\frac{1}{2} \lambda\{[\operatorname{sign}(\Re(\boldsymbol{w}))+i * \operatorname{sign}(\Im(\boldsymbol{w}))]\} .
$$

Finally, from (6), (7) and (12) the ZA-MP-CFxLMS algorithm can be derived as

$$
\begin{aligned}
\boldsymbol{w}(n+1)=\boldsymbol{w}(n) & -\frac{1}{2} \mu \boldsymbol{G}^{*} \boldsymbol{e} \\
& -\frac{1}{2} \mu \lambda[\operatorname{sign}(\Re(\boldsymbol{w}))+i * \operatorname{sign}(\Im(\boldsymbol{w}))] .
\end{aligned}
$$




\section{WAVE DOMAIN NOISE CANCELLATION USING THE ZA-WD-CFXLMS ALGORITHM}

In this section, we develop a zero-attracting complex FxLMS algorithm for wave domain noise cancellation.

The principal of wave domain signal representation is to use fundamental solutions of the Helmholtz wave equation as basis functions to express any wavefield over a spatial region. In this paper, we opt for the cylindrical harmonic based wave equation solution [30]. This solution decomposes any homogeneous incident wavefield $\rho(k)$ observed at $\left(r^{\prime}, \phi\right)$ into

$$
\rho\left(r^{\prime}, \phi, k\right)=\sum_{m=-\infty}^{\infty} a_{m}(k) J_{m}\left(k r^{\prime}\right) \exp (i m \phi)
$$

where $k=2 \pi f / C$ is the wave number, $f$ is the frequency, $C$ is the speed of sound, $a_{m}$ is the $m^{\text {th }}$ wave domain coefficient, and $J_{m}(\cdot)$ is the cylindrical Bessel function. Within this circular region, the infinite summation in (14) can be truncated at $M=\lceil e k r / 2\rceil$ [31], where $r$ is the radius of the region of interest. The transfer function in the wave domain could be represented by

$$
G\left(r^{\prime}, \phi, k\right)=\sum_{m=-M}^{M} g_{m}(k) J_{m}\left(k r^{\prime}\right) \exp (i m \phi)
$$

where $g_{m}(k)=\frac{i}{4} H_{m}^{(1)}(k\|\boldsymbol{z}\|)$.

Similar to the multi-point case, consider an adaptive ANC system using a $P$-point microphone array and a $Q$-point loudspeaker array in free field conditions. The observed residual signal $e$ of (1) and its decomposition can now be written in the wave domain following (14). Let the corresponding cylindrical harmonic coefficients be $\alpha_{m}, \beta_{m}$ and $\gamma_{m}$ respectively. The wave domain residual signal is then

$$
\boldsymbol{\alpha}(n)=\boldsymbol{\beta}(n)+\boldsymbol{\gamma}(n)
$$

where all three terms are $(2 N+1)$-long vectors in the complex domain. An FxLMS type formulation of (16) is

$$
\boldsymbol{\alpha}(n)=\boldsymbol{\beta}(n)+\operatorname{diag}(\boldsymbol{g}) \boldsymbol{w}(n),
$$

where $\boldsymbol{g}=\left[g_{-M}, \cdots, g_{M}\right]$. Note that $\boldsymbol{w}$ is now in the wave domain and therefore, can not be directly populated with loudspeaker weights $\boldsymbol{d}$ as before. Therefore, we need to form an appropriate translation relationship of the form $\boldsymbol{w}=\boldsymbol{T d}$, where $\boldsymbol{T}$ is the transformation matrix. For this purpose, we utilize the cylindrical harmonic decomposition of a $2 \mathrm{D}$ point source [32], and define $\boldsymbol{T}$ as

$$
\boldsymbol{T}=\left[\begin{array}{ccc}
e^{i M \phi_{1}} & \ldots & e^{i M \phi_{Q}} \\
\vdots & \ddots & \vdots \\
e^{-i M \phi_{1}} & \ldots & e^{-i M \phi_{Q}}
\end{array}\right]
$$

To calculate the updated equation (5) in wave domain, the gradient term in (5) can be written as

$$
\nabla \boldsymbol{\xi}(n)=\underbrace{\nabla \frac{1}{2} \boldsymbol{\alpha}(n)^{2}}_{\nabla \boldsymbol{\xi}_{1}}+\underbrace{\nabla \lambda\|\boldsymbol{d}(n)\|_{1}}_{\nabla \boldsymbol{\xi}_{2}} .
$$

From the wave domain Filtered-X Least Mean Square algorithm [20], the term $\nabla \boldsymbol{\xi}_{1}$ of (19) is simply $\nabla \boldsymbol{\xi}_{1}=(\operatorname{diag}(\boldsymbol{g}))^{*} \boldsymbol{\alpha}$. Since each items in (10) in wave domain are,

$$
\frac{\partial\|\Re(\boldsymbol{d})\|_{1}}{\partial \boldsymbol{x}}=\Re\left(\left(\boldsymbol{T}^{-1}\right)^{T}\right) * \operatorname{sign}(\Re(\boldsymbol{d}))
$$

$$
\begin{gathered}
\frac{\partial\|\Re(\boldsymbol{d})\|_{1}}{\partial \boldsymbol{y}}=-\Im\left(\left(\boldsymbol{T}^{-1}\right)^{T}\right) * \operatorname{sign}(\Re(\boldsymbol{d})) \\
\frac{\partial\|\Im(\boldsymbol{d})\|_{1}}{\partial \boldsymbol{x}}=\Im\left(\left(\boldsymbol{T}^{-1}\right)^{T}\right) * \operatorname{sign}(\Im(\boldsymbol{d})) \\
\frac{\partial\|\Im(\boldsymbol{d})\|_{1}}{\partial \boldsymbol{y}}=\Re\left(\left(\boldsymbol{T}^{-1}\right)^{T}\right) * \operatorname{sign}(\Im(\boldsymbol{d})),
\end{gathered}
$$

Substituting (20) into (9), $\partial \boldsymbol{\xi}_{2} / \partial \boldsymbol{w}^{*}$ becomes

$$
\begin{aligned}
\frac{\partial \xi_{2}}{\partial \boldsymbol{w}^{*}}=\frac{1}{2} \lambda\left\{\left[\Re\left(\left(\boldsymbol{T}^{-1}\right)^{T}\right)-i *\right.\right. & \left.\Im\left(\left(\boldsymbol{T}^{-1}\right)^{T}\right)\right] *\left[\operatorname{sign}\left(\Re\left(\boldsymbol{T}^{-1} \boldsymbol{w}\right)\right)\right. \\
& \left.\left.+i * \operatorname{sign}\left(\Im\left(\boldsymbol{T}^{-1} \boldsymbol{w}\right)\right)\right]\right\} .
\end{aligned}
$$

Thus, the ZA-WD-CFxLMS algorithm can be derived as

$$
\begin{aligned}
& \boldsymbol{w}(n+1)=\boldsymbol{w}(n)-\frac{1}{2} \mu(\operatorname{diag}(\boldsymbol{g}))^{*} \boldsymbol{\alpha} \\
& -\frac{1}{2} \mu \lambda\left\{[ \Re ( ( \boldsymbol { T } ^ { - 1 } ) ^ { T } ) - i * \Im ( ( \boldsymbol { T } ^ { - 1 } ) ^ { T } ) ] * \left[\operatorname{sign}\left(\Re\left(\boldsymbol{T}^{-1} \boldsymbol{w}\right)\right)\right.\right. \\
& \left.\left.\quad+i * \operatorname{sign}\left(\Im\left(\boldsymbol{T}^{-1} \boldsymbol{w}\right)\right)\right]\right\} . \quad
\end{aligned}
$$

\section{SIMULATION}

In this section, the proposed ZA-MP-CFxLMS and ZA-WDCFxLMS algorithms are evaluated. The sparse noise field is assumed to be a $200 \mathrm{~Hz}$ wavefield arriving from a $2 \mathrm{D}$ point source located at a radius of $2.5 \mathrm{~m}$ along the positive $\mathrm{x}$-axis. Noise cancellation over a $2 \mathrm{D}$ spatial region of radius $1 \mathrm{~m}$ is observed.

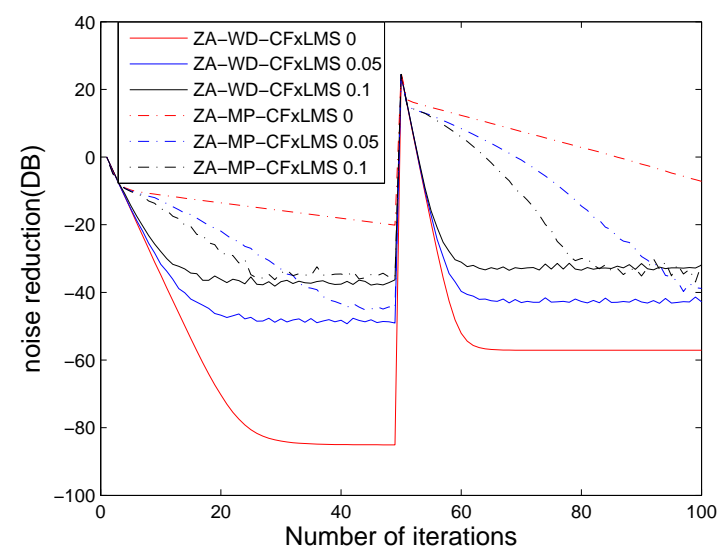

Fig. 1. Comparison of convergence performance for noise cancellation using ZA-WD-CFxLMS and ZA-MP-CFxLMS algorithm for variable zero attractor strength $(\lambda=0,0.05,0.1)$.

In the feedback control system, we use a circular microphone array of radius $1 \mathrm{~m}$ and circular loudspeaker array of radius $2 \mathrm{~m}$, both of which are equi-angularly spaced. The microphone array also coincides with the boundary of the quiet zone. A signal-to-noise (SNR) ratio of $40 \mathrm{~dB}$ is added at each microphone. According to the rule of thumb [31], in the wave domain, such a noise field within the desired quiet zone (radius of $1 \mathrm{~m}$ ) needs $m \in[-5,5]$ orthogonal modes to represent. Thus, we place 11 loudspeakers and 11 microphones in each corresponding array. In order to evaluate the noise cancellation levels over the spatial control region, we average the residual signal energy over 1296 points uniformly placed inside the region. 


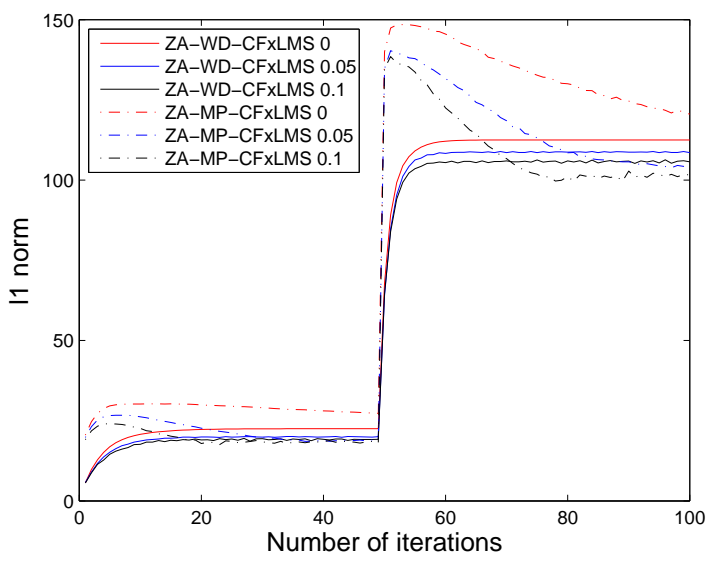

Fig. 2. Comparison of convergence performance for $\ell_{1}$-norm of the loudspeaker weights using ZA-WD-CFxLMS and ZA-MP-CFxLMS algorithm for variable zero attractor strength $(\lambda=0,0.05,0.1)$.

Plots in Fig. 1 show the comparison of the proposed algorithm's convergence performance vs spatial noise reduction. Plots in Fig. 2 show the comparison of the convergence performance vs the $\ell_{1}$ norm constraint. The results in Fig. 3 demonstrate the spatial residual signal energy over the entire control region for the proposed algorithms. Below is a case by case analysis of the results shown in the above figures.

Case 1-Multi-point sparse field ANC: The dotted lines in Fig. 1 compare the convergence performance of ZA-MP-CFxLMS in the time-varying noise field. Compared to the dotted red line with $\lambda=0$ (which is the conventional MP-FxLMS [17]), the convergence speed of its $\ell_{1}$ constrained variants (blue and black) are significantly faster. A general observation is that as $\lambda$ increases, the convergence performance gets better. At the 50th iteration we forcefully increase the noise power, and the corresponding re-convergence time for ZAMP-CFxLMS again decreases with increasing $\lambda$. From Fig. 2, we can see that as $\lambda$ increases, the $\ell_{1}$-norm of loudspeaker weights decreases and produces less energy. From Fig.3(b) and Fig.3(c), we observe that introduction of the $\ell_{1}$ constraint causes better noise cancellation performance after the same 30 iterations. This is a significant advantage in practical noise applications, which are constantly time-varying.

Case 2-Wave domain sparse field ANC: The solid lines in Fig. 1 show the convergence performance of ZA-WD-CFxLMS in the time-varying noise field. As we expected, the convergence speed of ZA-WD-CFxLMS algorithms is much faster than ZA-MPCFxLMS in for all values of $\lambda$. For each $\lambda$, the ZA-WD-CFxLMS demonstrates more noise reduction and faster convergence so it can track the variation of the noise field and achieve better performance than the ZA-MP-CFxLMS. With the sparse constraint added in the FxLMS in wave domain, the total energy of loudspeaker weights can be reduced as shown in Fig 2. Fig 3(d) shows a significant noise reduction in the designed region after 30 iterations of ZAWD-CFxLMS. One drawback is, compared to the solid red line with $\lambda=0$ in Fig. 1 (which is the conventional WD-FxLMS [20]), adding the sparse constraint decreases the noise reduction level of the ANC system (blue and black solid line in Fig. 1).

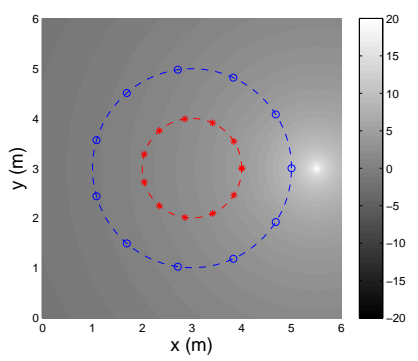

(a)

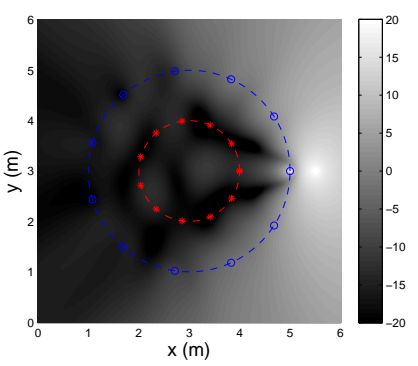

(c)

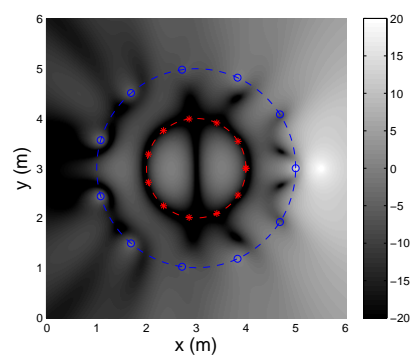

(b)

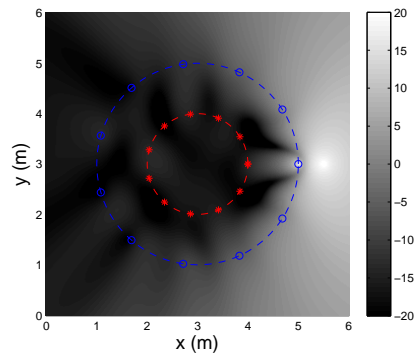

(d)
Fig. 3. The results of ANC in free-field. The inner array is a microphone array and the outer array is a loudspeaker array. (a) The energy of the initial sparse noise field. (b) The residual energy after 30 iterations of MP-FxLMS. (c) The residual energy after 30 iterations of ZA-MP-CFxLMS. (d) The residual energy after 30 iterations of ZA-WD-CFxLMS.

\section{CONCLUSION}

We introduce the compressive sampling into the adaptive processing and propose ZA-MP-CFxLMS and ZA-WD-CFxLMS algorithm for active noise control over spatial region. We compare the performance of the two new algorithms with the conventional multichannel algorithms in the feedback ANC system. In the sparsely distributed noise field, the multi-point sparse complex FxLMS algorithm can achieve better adaptive performance than the MP-FxLMS algorithm. For the wave domain complex FxLMS algorithm, introducing the sparse constraint can reduce the total energy of the loudspeaker weights, but there exits a trade off between noise reduction and the energy of the loudspeaker weights. At the same time, wave domain sparse FxLMS achieves significant noise reduction over the entire designed region with fast convergence speed, which can track the variation of the noise field very well.

\section{REFERENCES}

[1] S. M. Kuo and D. R. Morgan, Active Noise Control Systems: Algorithms and DSP Implementations, Wiley, New York, 1996.

[2] M. Bergamasco, F. D. Rossa, and L. Piroddi, "Active noise control with on-line estimation of non-Gaussian noise characteristics," Journal of Sound and Vibration, vol. 331, no. 1, pp. 27 - 40, January 2012.

[3] C. C. Fuller, S. Elliott, and P. A. Nelson, Active Control of Vibration, Academic, San Diego, CA, 1996.

[4] J.D. McIntosh, "Active noise cancellation aircraft headset system," August 2001, US Patent 6,278,786. 
[5] S. M. Kuo, S. Mitra, and W. S. Gan, "Active noise control system for headphone applications," IEEE Transactions on Control Systems Technology, vol. 14, no. 2, pp. 331-335, March 2006.

[6] S.M. Kuo, X. Kong, and W.S. Gan, "Applications of adaptive feedback active noise control system," IEEE Transactions on Control Systems Technology, vol. 11, no. 2, pp. 216-220, March 2003.

[7] T. J. Sutton, S. J. Elliott, A. M. McDonald, and T. J. Saunders, "Active control of road noise inside vehicles," Noise Control Engineering Journal, vol. 42, no. 4, pp. 9-12, July 1994.

[8] H. Sano, T. Inoue, A. Takahashi, K. Terai, and Y. Nakamura, "Active control system for low-frequency road noise combined with an audio system," IEEE Transactions on Speech and Audio Processing, vol. 9, no. 7, pp. 755-763, October 2001.

[9] J. Cheer and S. J. Elliott, "The design and performance of feedback controllers for the attenuation of road noise in vehicles," International Journal of Acoustics and Vibration, vol. 19, no. 3, pp. 155-164, January 2014.

[10] J. C. Burgess, "Active adaptive sound control in a duct: A computer simulation," The Journal of the Acoustical Society of America, vol. 70, no. 3, pp. 715-726, June 1981.

[11] E. A. Wan, "Adjoint LMS: an efficient alternative to the filtered-x LMS and multiple error LMS algorithms," in Proc. IEEE International Conference on Acoustics, Speech, and Signal Processing 1996, Atlanta, Georgia, USA, May 1996, vol. 3, pp. 1842-1845.

[12] S. M. Kuo and D. R. Morgan, "Active noise control: A tutorial review," Proceedings of the IEEE, vol. 87, no. 6, pp. 943-973, June 1999.

[13] S. J. Elliott, I. Stothers, and P. A. Nelson, "A multiple error LMS algorithm and its application to the active control of sound and vibration," IEEE Transactions on Acoustics, Speech and Signal Processing, vol. 35, no. 10, pp. 1423-1434, October 1987.

[14] S. Spors and H. Buchner, "Efficient massive multichannel active noise control using wave-domain adaptive filtering," in Proc. 3rd International Symposium on Communications, Control and Signal Processing, St. Julians, Malta, March 2008, pp. 1480-1485.

[15] S. J. Elliot, P. A. Nelson, I. M. Stothers, and C. C. Boucher, "Inflight experiments on the active control of propeller-induced cabin noise," Journal of Sound and Vibration, vol. 140, no. 2, pp. 219-238, September 1990.

[16] J. Lorente, M. Ferrer, M. De Diego, and A. González, "GPU implementation of multichannel adaptive algorithms for local active noise control," IEEE/ACM Transactions on Audio, Speech, and Language Processing, vol. 22, no. 11, pp. 16241635, November 2014.

[17] T. Kosaka, S. J. Elliott, and C. C. Boucher, "A novel frequency domain filtered-x LMS algorithm for active noise reduction," in Proc. IEEE International Conference on Acoustics, Speech, and Signal Processing 1997, Munich, Germany, April 1997, vol. 1, pp. 403-406.

[18] Y. J. Wu and T. D. Abhayapala, "Soundfield reproduction using theoretical continuous loudspeaker," in Proc. IEEE International Conference on Acoustics, Speech, and Signal Processing 2008, Las Vegas, Nevada, USA, March 2008, pp. 377-380.
[19] Y.J. Wu and T.D. Abhayapala, "Theory and design of soundfield reproduction using continuous loudspeaker concept," IEEE Transactions on Audio, Speech, and Language Processing, vol. 17, no. 1, pp. 107-116, January 2009.

[20] J. Zhang, W. Zhang, and T. D. Abhayapala, "Noise cancellation over spatial regions using adaptive wave domain processing," in Proc. IEEE Workshop on Applications of Signal Processing to Audio and Acoustics (WASPAA), New Paltz, New York, USA, October 2015, pp. 1-5.

[21] H. Chen, P. Samarasinghe, T.D. Abhayapala, and W. Zhang, "Spatial noise cancellation inside cars: Performance analysis and experimental results," in Proc. IEEE Workshop on Applications of Signal Processing to Audio and Acoustics (WASPAA), New Paltz, New York, USA, October 2015, pp. 1-5.

[22] H. Chen, P. Samarasinghe, and T.D. Abhayapala, "In-car noise field analysis and multi-zone noise cancellation quality estimation," in Proc. Asia-Pacific Signal and Information Processing Association Annual Summit and Conference (APSIPA), December 2015.

[23] J. F. de Andrade Jr., M. L. R. de Campos, and J. A. Apolinario Jr., "An L1-constrained normalized LMS algorithm and its application to thinned adaptive antenna arrays," in Proc. IEEE International Conference on Acoustics, Speech, and Signal Processing 2013, Vancouver, British Columbia, Canada, May 2013, pp. 3806-3810.

[24] Y. Chen, Y. Gu, and A. O. Hero, "Sparse LMS for system identification," in Proc. IEEE International Conference on Acoustics, Speech, and Signal Processing 2009, Taipei, Taiwan, April 2009, pp. 3125-3128.

[25] K. Shi and X. Ma, "Transform domain LMS algorithms for sparse system identification," in Proc. International Conference on Acoustics Speech and Signal Processing 2010, Dallas, Texas, USA, March 2010, pp. 3714-3717.

[26] J. Yang and G. E. Sobelman, "Sparse LMS with segment zero attractors for adaptive estimation of sparse signals," in Proc. IEEE Asia Pacific Conference on Circuits and Systems (APCCAS) 2010, Kuala Lumpur, Malaysia, December 2010, pp. 422-425.

[27] B. Widrow, J. McCool, and M. Ball, "The complex LMS algorithm," Proceedings of the IEEE, vol. 63, no. 4, pp. 719-720, April 1975.

[28] S. Haykin, Adaptive Filter Theory, Prentice Hall, 2001.

[29] D. Sarason, Complex Function Theory, American Mathematical Soc., 2007.

[30] D.B. Ward and T.D. Abhayapala, "Reproduction of a planewave sound field using an array of loudspeakers," IEEE Transactions on Speech and Audio Processing, vol. 9, no. 6, pp. 697707, September 2001.

[31] R. A. Kennedy, P. Sadeghi, T. D. Abhayapala, and H. M. Jones, "Intrinsic limits of dimensionality and richness in random multipath fields," IEEE Transactions on Signal Processing, vol. 55, no. 6, pp. 2542-2556, June 2007.

[32] G. Williams, Fourier Acoustics: Sound Radiation and Nearfield Acoustical Holography, Academic, New York, NY, USA, 1999. 